\title{
Prevalência de violência e fatores associados em idosos de unidades de saúde em uma capital da Amazônia ocidental
}

\author{
Prevalence of violence and factors associated in elderly health units in a capital of the \\ western Amazon
}
Prevalencia de violencia y factores asociados en ancianos de unidades de salud en un capital de la Amazonia occidental

Polyana Caroline de Lima Bezerra ${ }^{1 *}$, Cleane Araújo Sampaio.

\section{RESUMO}

Objetivo: Identificar a prevalência de violência e a sua associação com variáveis socioeconômicas, demográficas e de saúde em idosos de Unidades de Atenção Primária à Saúde em um Estado do Norte do Brasil. Métodos: Trata-se de um estudo transversal com 290 idosos acompanhados na atenção primária à saúde na Capital do Acre, no período 2016-2017. Identificou-se as prevalências de cada tipo de violência e verificada a prevalência da violência geral. Por meio da Regressão Poisson, testaram-se os fatores associados à violência geral, com Razões de Prevalência Bruta (RP) com seus respectivos intervalos de confiança $\left(\mathrm{IC}_{95 \%}\right)$. Após regressão bivariada, foi realizada a análise multivariada. Permaneceram no modelo final apenas aquelas que apresentaram melhor ajuste nas razões de prevalência e $p \leq 0,05$. Resultados: $A$ violência psicológica foi mais prevalente na amostra (30,6\%). As variáveis segurança de bairro, renda família, síndrome de fragilidade, depressão, câncer, acidente vascular cerebral, raça/cor e excesso de bebida alcóolica, foram os fatores associados à prevalência de violência geral. Conclusão: evidenciou-se alta a prevalência de violência nos idosos estudados e os fatores associados foram, majoritariamente, relacionados à uma saúde fragilizada. Defende-se que estratégias de promoção do envelhecimento saudável possam também auxiliar no enfrentamento da violência contra a população idosa.

Palavras-chave: Idoso, Maus-tratos ao idoso, Prevalência.

\begin{abstract}
Objective: To identify the prevalence of violence and its association with socioeconomic, demographic and health variables in elderly people from Primary Health Care Units in a state in northern Brazil. Methods: This is a cross-sectional study with 290 elderly people followed up in primary health care in the capital of Acre, in the period 2016-2017. The prevalence of each type of violence was identified and the prevalence of general violence was verified. Through Poisson Regression, the factors associated with general violence were tested, with Gross Prevalence Ratios (PR) with their respective confidence intervals $(95 \% \mathrm{Cl})$. After bivariate regression, multivariate analysis was performed. Only those that showed the best fit in the prevalence ratios and $p \leq 0.05$ remained in the final model. Results: Psychological violence was more prevalent in the sample $(30.6 \%)$. The variables neighborhood security, family income, fragility syndrome, depression, cancer, stroke, race / color and excess of alcoholic beverages, were the factors associated with the prevalence of general violence. Conclusion: there was a high prevalence of violence in the elderly studied and the associated factors were mostly related to weakened health. It is argued that strategies to promote healthy aging can also assist in tackling violence against the elderly population.
\end{abstract}

Keywords: Elderly, Elder abuse, Prevalence.

1 Universidade Federal do Acre (UFAC), Rio Branco - AC.

*E-mail: polyana@ufac.br 


\section{RESUMEN}

Objetivo: Identificar la prevalencia de la violencia y su asociación con variables socioeconómicas, demográficas y de salud en personas mayores de las Unidades de Atención Primaria de Salud en un estado en el norte de Brasil. Métodos: Este es un estudio transversal con 290 personas mayores seguidas en atención primaria de salud en la capital de Acre, en el período 2016-2017. Se identificó la prevalencia de cada tipo de violencia y se verificó la prevalencia de la violencia general. A través de la regresión de Poisson, se probaron los factores asociados con la violencia general, con razones de prevalencia bruta (RP) con sus respectivos intervalos de confianza (IC 95\%). Después de la regresión bivariada, se realizó un análisis multivariado. Solo aquellos que mostraron el mejor ajuste en las razones de prevalencia y $p \leq 0.05$ permanecieron en el modelo final. Resultados: La violencia psicológica fue más prevalente en la muestra $(30,6 \%)$. Las variables seguridad del vecindario, ingresos familiares, síndrome de fragilidad, depresión, cáncer, accidente cerebrovascular, raza / color y exceso de bebidas alcohólicas, fueron los factores asociados con la prevalencia de violencia general. Conclusión: hubo una alta prevalencia de violencia en los ancianos estudiados y los factores asociados se relacionaron principalmente con la salud debilitada. Se argumenta que las estrategias para promover un envejecimiento saludable también pueden ayudar a combatir la violencia contra la población de edad avanza.

Palabras clave: Anciano, Maltrato al anciano, Prevalencia.

\section{INTRODUÇÃO}

O envelhecimento populacional é crescente e vem acontecendo mundialmente. Estatísticas apontam que em 2025 o Brasil será o sexto país com a população mais envelhecida do mundo (CHAIMOWICZ F, 1997). Casos de violência contra a pessoa idosa não são raros. Uma revisão sistemática com dados de 28 países, em 2017, revelou que $15,7 \%$ das pessoas com idade igual ou superior a 60 anos sofreram algum tipo de violência no último ano (YON Y, et al., 2017).

A Organização Mundial da Saúde (OMS) classifica cinco tipos de violência a) violência física - quando existe a imposição de dor ou lesão, coação física, ou domínio induzido pela força ou por substâncias psicoativas; b) abuso psicológico ou emocional - que causa angustia mental a pessoa idosa; c) violência financeira ou material - que é caracterizada pela exploração indevida e também por utilizar recursos financeiros; d) violência sexual - contato de qualquer natureza que não seja consensual com pessoas idosas; e) negligência - caracteriza-se pela rejeição ou pelo fracasso da família, em se dedicar a obrigação em cuidar do idoso. A negligencia ao idoso pode ser intencional ou não, e causar dor física ou emocional ao idoso (KRUG EG, et al., 2002).

A violência contra a pessoa idosa além de contribuir para o aumento de doenças e interferir na qualidade vida do indivíduo, causa prejuízos aos cofres públicos, aumentando os gastos com a saúde; reduzindo os anos de vida saudável e aumentando o número de mortes, por complicações advindas da violência.

Pode-se afirmar que a violência gera consequências irreparáveis, implicando em danos materiais, morais e que podem levar ao óbito (SILVA CFS e DIAS CMSB, 2016). O referido estudo tem como objetivo identificar a prevalência da violência geral em idosos acompanhados na atenção primária à saúde na capital do Estado do Acre, e verificar quais os fatores sociodemográficos, os hábitos de vida e condições de saúde que estão associados à essa condição.

\section{MÉTODOS}

Trata-se de um estudo transversal com idosos de duas Unidades de Saúde (US) do Acre, cujo trabalho de campo foi realizado de outubro de 2016 a junho de 2017. Refere-se a um subprojeto que utilizou parte do banco de dados de uma pesquisa matriz denominada "Fragilidade e condições de saúde de idosos em Rio Branco, Acre".

O projeto matriz foi submetido e aprovado pelo Comitê de Ética em Pesquisa da Escola Nacional de Saúde Pública Sergio Arouca - ENSP/ FIOCRUZ (CAAE n 58791716.5.0000.5240) e os aspectos éticos seguiram 
as recomendações da Resolução ํo. 466/12, do Conselho Nacional de Saúde do Ministério da Saúde (BRASIL, 2013).

A amostra da pesquisa foi por conglomerado em dois estágios: unidades de saúde e os indivíduos idosos. O projeto matriz selecionou uma Unidade de Referência em Atenção Primária à Saúde e uma Unidade de Saúde da Família, localizadas em duas regionais de saúde distintas.

Os critérios para seleção das unidades de saúde foram o maior número de idosos cadastrados e estarem com o cadastro das famílias atualizado. Portanto, a escolha das duas unidades de saúde se deu em virtude do quantitativo de idosos por elas acompanhados. Foram considerados elegíveis para o estudo, indivíduos com idade igual ou superior a 60 anos, de ambos os sexos, que residiam na área adscrita e com cadastro nas unidades selecionadas.

Foram critérios de exclusão, os idosos institucionalizados e aqueles com situação de saúde que inviabilizava a participação, incluindo os que possuíam diagnóstico de doenças cognitivas que o impossibilitava de responder por si.

Foram consideradas perdas, os casos em que os sujeitos se negaram a responder o questionário, aqueles que não foram encontrados em domicílio após duas tentativas no local de residência sem sucesso ou por motivo de mudança, e os idosos que não possuíam todos os elementos necessários para classificação na escala de triagem para violência.

Para o tamanho da amostra, foi levado em consideração: o tamanho populacional de idosos das UBS selecionadas ( $\mathrm{N}=953)$, uma prevalência estimada de $10 \%$, um grau de confiança de $95 \%$ e um erro amostral de $3 \%$, com o acréscimo de $20 \%$ para possíveis perdas, resultando em uma amostra final estimada em 365 idosos. No entanto, após o procedimento dos critérios de exclusão e perdas, a amostra do referido estudo analisou 290 idosos.

A coleta de dados foi feita por meio de entrevistas realizadas por uma equipe comporta pela coordenadora da pesquisa e estudantes de medicina e de pós-graduação em ciência da saúde, em domicílio mediante anuência dos participantes após a assinatura do Termo de Consentimento Livre e Esclarecido. O instrumento utilizado foi o questionário validado do estudo EPIFLORIPA que contempla 13 blocos temáticos, referentes aos dados socioeconômicos e demográficos, de hábitos de vida e condições de saúde.

Dentro do referido questionário, foi aplicado o instrumento denominado Hwalek-Sengstock Elder Abuse Screening Test (H-S/EAST), do qual foram obtidos os dados para avaliar o desfecho de interesse do presente estudo. O H-S/EAST é validado no Brasil e recomendado para ser aplicado em Unidades de Saúde, por se tratar de um instrumento de triagem de violência (NEALES AV, et al., 1991; REICHENHEIM ME, et al., 2008).

De acordo com o H-S/EAST, classificou-se como violência psicológica as respostas afirmativas para as questões: "Alguma outra pessoa toma decisões sobre sua vida, do tipo como o(a) Sr.(a) deve viver ou onde deve morar?; "Alguém da sua família obriga o(a) Sr.(a) a ficar na cama ou the diz que está doente, quando o(a) Sr.(a) sabe que não está?" e "Alguém já obrigou o(a) Sr.(a) a fazer coisas que não queria fazer?; como violência financeira a resposta para "Alguém já pegou coisas que pertencem ao(a) Sr.(a) sem o seu consentimento?" e como violência física, a questão "Alguém próximo ao(a) Sr.(a) tentou machucá-lo(a) ou prejudicá-lo(a) recentemente. A violência geral, foi definida por meio da resposta "sim" em alguma das perguntas supracitadas.

A partir da revisão de literatura, foram selecionadas as seguintes variáveis exploratórias: sexo, idade (60 a 69 anos, 70 a 79 e 80 ou mais); cor da pele/etnia autorreferida (branca, não branca [parda, negra, amarela, indígena); cidade de nascimento (Rio Branco, outra cidade do Acre, outras cidades da Região Norte, outras cidades do Brasil); tem companheiro(a); vive sozinho, Renda familiar ( $\geq 3$ s.m; 1 a $<3$ s.m; $<1$ s.m) e segurança do bairro, por meio da resposta ao questionamento "durante o dia, o sr. (a) acha seguro caminhar, andar de bicicleta ou praticar esportes perto de sua casa?", a resposta sim/não convertida para a variável segurança do bairro satisfatória/insatisfatória.

As variáveis independentes referentes aos hábitos de vida e situação de saúde, foram verificadas por meio do hábito tabagista, analisado de forma dicotômica (não [não fuma/fumou e parou]e sim); o excesso de consumo de álcool foi verificado por meio do Alcohol Use Disorders Identification Test (AUDIT) (BABOR TF, 
et al. 2001); a prática de atividade física foi obtida pelo questionário International Physical Activity Questionnaire (IPAQ), identificando aqueles que praticavam atividade física pelo menos 3 vezes por semana (sim ou não) (MATSUDO SM, et al., 2001).

O risco nutricional foi de acordo com a Mini Avaliação Nutricional (MAN), que verificou o estado nutricional normal, risco de desnutrição e desnutrição; risco nutricional 2 classificados sem risco nutricional os que tinham (estado nutricional normal), em risco nutricional o que apresentaram (risco de desnutrição/desnutrição) (GUIDOZ Y, et al., 1994); o índice de Massa Corporal foi realizado pela medida de peso divide pela altura ao quadrado, segundo parâmetros para idosos pela OMS sendo classificados em Eutrófico 18,5 a 24,9, Baixo peso $<18,5$, Sobrepeso 25 a 29,9 e Obesidade 30 ou mais, (OMS, 1995).

A percepção de saúde por estratos (muito boa, boa, regular, ruim, muito ruim) e bivariada, como percepção de saúde satisfatória (sim/não); Percepção de saúde bucal (Satisfatória (sim/não); o déficit cognitivo foi investigado através do Mini Exame do Estado Mental (MEEM), utilizando o ponto de corte para idosos com baixa escolaridade (<8anos de estudo) 17 pontos e para aqueles com maior escolaridade ( $\geq 8$ anos de estudo) 25 pontos (BERTOLUCCI PHF, et al., 1994); a incapacidade funcional foi verificada pela Escala de Atividades Básicas e Instrumentais da Vida Diária (ROSA TEC, et al., 2003).

A história de queda no último ano (não ou sim); a polifamárcia foi analisada pelo número de medicamentos em uso (< 5 medicamentos [não] $/ \geq 5$ medicamentos [sim]); a depressão foi avaliada pela Geriatric Depression Scale (GDS-15) (ALMEIDA OP e ALMEIDA SA, 1999).

A verificação das morbidades autorreferidas pelos idosos foi dada pela listagem de morbidades que foram diagnosticados em algum momento da vida. Por seguinte, foi analisada de forma dicotômica (sim/não) e pelo somatório das afirmativas positivas na listagem das morbidades autorreferidas, categorizada em: nenhuma, uma a três, $\geq 4$ morbidades.

Semelhantemente, foram realizadas perguntas para verificar se os idosos tinham doenças de coluna (não ou sim), hipertensão (não ou sim), Reumatismo, artrite, artrose (não ou sim), Doença do coração/cardiovascular (não ou sim), osteoporose (não ou sim), depressão (não ou sim), diabetes (não ou sim), câncer (não ou sim). A síndrome de fragilidade foi identificada pela Edmonton Frail Scale (EFS) (ROLFSON DB, et al., 2006).

Foram calculadas as prevalências de cada tipo de violência e verificada a prevalência da violência geral, segundo as categorias das variáveis independentes. Em seguida, por meio da Regressão Poisson, testaramse os fatores associados à violência geral.

Assim, obtiveram-se as Razões de Prevalência Bruta (RP) nas análises bivariada e Prevalência Ajustada na análise multivariável com seus respectivos intervalos de confiança $\left(\mathrm{IC}_{95} \%\right)$. A partir dos resultados da regressão bivariada, foram incluídas na análise multivariável todas as variáveis que apresentaram $p \leq 0,20$. Permaneceram no modelo final apenas aquelas que apresentaram melhor ajuste nas RP e $p \leq 0,05$.

Todas as análises estatísticas foram realizadas considerando-se o efeito do desenho amostral e incorporando-se os pesos amostrais e foram conduzidas no programa estatístico Statistical Package for Social Sciences Statistic (SPSS) Data 20 (Chicago, IL, 2008).

\section{RESULTADOS}

A prevalência de violência direta, identificada pelo instrumento utilizado na amostra, foi de $52,6 \%$. 0 Gráfico 1 apresenta os percentuais segundo os tipos de violência. A violência mais relatada foi a psicológica $(n=88 ; 30,6 \%)$, seguida da financeira $(n=72 ; 25,0 \%)$ e física $(n=49 ; 17,2 \%)$. 
Gráfico 1 - Prevalência de violência, segundo o tipo. Rio Branco, Acre. Idosos, $2016-2017$.

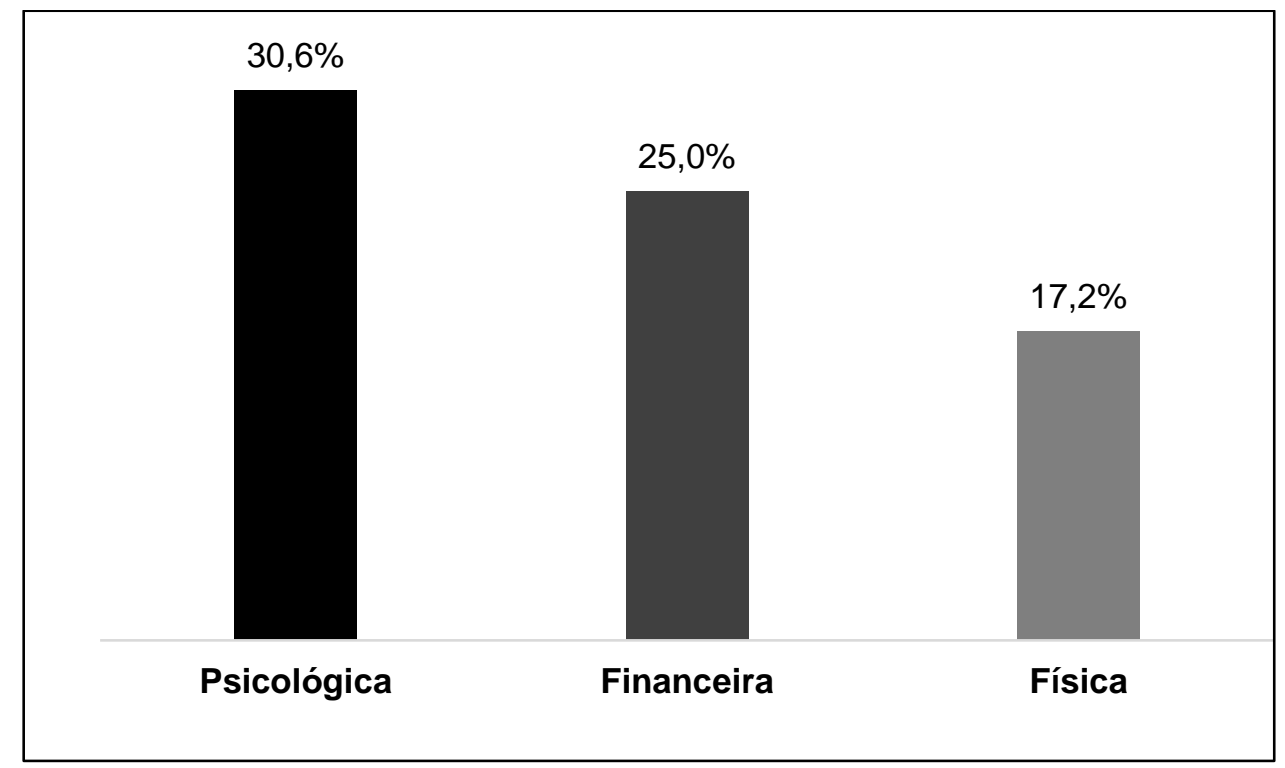

Fonte: Bezerra PCL e Sampaio CA, 2020.

Constatou-se que a maioria dos relatos de violência era do sexo feminino $(308 ; 63 \%)$. Da mesma maneira a violência foi mais prevalente em idosos que não viviam sozinhos $(441 ; 90,2 \%)$, que não fazem uso em excesso de bebida alcóolica (450; 92,2), com risco nutricional (246; 60\%), com percepção de saúde insatisfatória $(318 ; 65,8 \%)$, com percepção de saúde bucal satisfatória (260; $55,1 \%)$, que não tem déficit cognitivo (270; 56,7\%), que tem hipertensão (356; 73,3\%) (Tabela 1).

A Tabela 2 apresenta a análise bivariada observou-se que idosos com faixa etária $\geq 80$ anos tem 1,23 (IC95\%:1,06 - 1,42), de maior prevalência de sofrer violência comparado com idosos de outras faixas etárias. Os que se autodeclararam como raça/cor não branca tem 0,81 (IC95\%:0,70 - 0,92) de prevalência de sofrer violência sobre os da raça/cor branca. Os idosos que nasceram em outras cidades do Brasil apresentaram maior prevalência no desfecho 1,30 (IC95\%:1,05 - 1,60). Idosos com companheiros tem maior prevalência de sofre violência 1,15 (IC95\% 1,03-1,30). Os com escolarização de $1-3$ anos apresentaram maior prevalência de sofrer violência 1,73 (IC95\%:1,07 - 2,80). Aqueles com renda familiar maior que três salaries mínimos apresentaram maior prevalência de sofrer violência 1,71 (IC95\% 1,22-0,02). Aqueles que consideraram a segurança de bairro insatisfatória apresentaram maior prevalência de sofrer violência 1,57 (IC95\% 1,22-2,02). 


\section{Revista Eletrônica Acervo Saúde / Electronic Journal Collection Health ～ISSN 2178-2091}

Tabela 1 - Caracterização sociodemográfica, do estilo de vida e de saúde da amostra, segundo o risco de violência. Rio Branco, Acre. Idosos, 2016 - 2017.

\begin{tabular}{|c|c|c|c|c|c|c|c|c|c|}
\hline \multirow{3}{*}{ Variáveis $^{a}$} & \multirow{2}{*}{\multicolumn{2}{|c|}{ Total }} & \multicolumn{6}{|c|}{ Violência } & \multirow[t]{3}{*}{ p-valor } \\
\hline & & & \multicolumn{3}{|c|}{ Sim } & \multicolumn{3}{|c|}{ Não } & \\
\hline & n 290 & $N \exp 928$ & N 153 & $N \exp 334$ & $\%$ & N 195 & $N \exp 619$ & $\%$ & \\
\hline \multicolumn{10}{|l|}{ Sexo } \\
\hline Masculino & 112 & 347 & 60 & 151 & 36,8 & 52 & 196 & 37,8 & \multirow{2}{*}{0,785} \\
\hline Feminino & 178 & 581 & 93 & 259 & 63,2 & 85 & 322 & 62,2 & \\
\hline \multicolumn{10}{|l|}{ Faixa etária } \\
\hline 60 a 69 anos & 138 & 440 & 67 & 186 & 45,3 & 71 & 254 & 48,9 & \multirow{3}{*}{0,036} \\
\hline 70 a 79 anos & 100 & 313 & 54 & 131 & 31,9 & 46 & 181 & 35,0 & \\
\hline$\geq 80$ anos & 52 & 177 & 32 & 94 & 22,9 & 20 & 83 & 94,0 & \\
\hline \multicolumn{10}{|l|}{ Raça/cor autodeclarada } \\
\hline Branca & 46 & 148 & 29 & 79 & 19,3 & 17 & 69 & 13,3 & \multirow{2}{*}{0,009} \\
\hline Não branca & 244 & 781 & 124 & 331 & 80,7 & 120 & 450 & 86,7 & \\
\hline \multicolumn{10}{|l|}{ Cidade de nascimento } \\
\hline Rio Branco & 64 & 198 & 27 & 91 & 18,6 & 34 & 99 & 22,5 & \multirow{4}{*}{0,058} \\
\hline Outra cidade do Acre & 160 & 523 & 88 & 281 & 57,5 & 70 & 234 & 53,2 & \\
\hline Outras cidades da Reg. Norte & 43 & 130 & 19 & 57 & 11,7 & 22 & 68 & 15,5 & \\
\hline Outras cidades do Brasil & 31 & 101 & 19 & 60 & 12,3 & 11 & 39 & 8,9 & \\
\hline \multicolumn{10}{|l|}{ Com companheiro } \\
\hline $\operatorname{Sim}$ & 145 & 451 & 72 & 53 & 10,9 & 75 & 31 & 7,0 & \multirow{2}{*}{0,040} \\
\hline Não & 153 & 502 & 14 & 234 & 53,2 & 8 & 226 & 46,3 & \\
\hline \multicolumn{10}{|l|}{ Vive sozinho } \\
\hline Não & 269 & 860 & 136 & 441 & 90,2 & 125 & 395 & 91,2 & \multirow{2}{*}{0,460} \\
\hline Sim & 29 & 93 & 17 & 48 & 9,8 & 12 & 45 & 10,2 & \\
\hline \multicolumn{10}{|l|}{ Escolaridade } \\
\hline 11 ou mais & 12 & 34 & 4 & 11 & 2,3 & 5 & 23 & 5,2 & \multirow{5}{*}{0,010} \\
\hline $8-10$ anos & 31 & 101 & 15 & 46 & 9,5 & 16 & 55 & 12,5 & \\
\hline $4-7$ anos & 73 & 245 & 34 & 119 & 24,5 & 39 & 126 & 28,7 & \\
\hline $1-3$ anos & 57 & 160 & 32 & 91 & 18,7 & 24 & 66 & 15,0 & \\
\hline Zero & 125 & 405 & 67 & 219 & 45,1 & 53 & 169 & 38,5 & \\
\hline
\end{tabular}

REAS/EJCH | Vol.12(8) | e3434 | DOI: https://doi.org/10.25248/reas.e3434.2020 Página 6 de 17 


\begin{tabular}{|c|c|c|c|c|c|c|c|c|c|}
\hline Renda familia & & & & & & & & & \\
\hline$\geq 3$ s.m & 9 & 34 & 8 & 28 & 5,7 & 1 & 6 & 1,4 & \multirow{3}{*}{0,000} \\
\hline $1 \mathrm{a}<3 \mathrm{~s} . \mathrm{m}$ & 72 & 242 & 41 & 138 & 28,2 & 28 & 94 & 21,4 & \\
\hline$<1$ s.m & 217 & 677 & 104 & 340 & 77,3 & 108 & 323 & 66,1 & \\
\hline \multicolumn{10}{|c|}{ Segurança do bairro } \\
\hline Satisfatória & 187 & 122 & 9 & 40 & 8,3 & 20 & 73 & 16,7 & \multirow{2}{*}{0,013} \\
\hline Insatisfatória & 105 & 818 & 143 & 442 & 91,7 & 116 & 365 & 85,3 & \\
\hline \multicolumn{10}{|c|}{ Hábito tabagista } \\
\hline Não & 94 & 277 & 45 & 135 & 27,7 & 47 & 140 & 31,8 & \multirow{2}{*}{0,031} \\
\hline $\operatorname{Sim}$ & 17 & 664 & 108 & 84 & 17,2 & 90 & 95 & 21,6 & \\
\hline \multicolumn{10}{|c|}{ Excesso de bebida alcóolica ${ }^{b}$} \\
\hline Não & 278 & 883 & 141 & 450 & 92,2 & 131 & 414 & 95,6 & \multirow{2}{*}{0,260} \\
\hline Sim & 20 & 70 & 12 & 38 & 7,8 & 6 & 26 & 4,4 & \\
\hline \multicolumn{10}{|c|}{ Prática de atividade física $^{c}$} \\
\hline Sim & 64 & 205 & 7 & 25 & 5,1 & 4 & 14 & 3,2 & \multirow{2}{*}{0,000} \\
\hline Não & 234 & 748 & 56 & 174 & 35,6 & 25 & 76 & 17,2 & \\
\hline \multicolumn{10}{|c|}{ Risco nutricional $^{\mathrm{d}}$} \\
\hline Não & 96 & 285 & 56 & 164 & 40,0 & 40 & 122 & 38,5 & \multirow{2}{*}{0,607} \\
\hline Sim & 146 & 460 & 77 & 246 & 60,0 & 64 & 198 & 61,5 & \\
\hline \multicolumn{10}{|l|}{ IMCe } \\
\hline Eutrófico & 92 & 33 & 6 & 19 & 4,6 & 3 & 11 & 3,4 & \multirow{4}{*}{0,016} \\
\hline Baixo peso & 14 & 33 & 7 & 22 & 5,4 & 2 & 5 & 1,6 & \\
\hline Sobrepeso & 87 & 61 & 9 & 27 & 6,6 & 12 & 32 & 10 & \\
\hline Obeso & 66 & 619 & 111 & 342 & 83,4 & 87 & 272 & 85,0 & \\
\hline \multicolumn{10}{|c|}{ Estado de saúde autorreferida } \\
\hline Muito bom & 12 & 38 & 3 & 8 & 1,7 & 8 & 28 & 6,4 & \multirow{5}{*}{0,000} \\
\hline Bom & 96 & 292 & 52 & 157 & 32,5 & 44 & 136 & 30,9 & \\
\hline Regular & 131 & 424 & 66 & 206 & 42,7 & 44 & 213 & 48,4 & \\
\hline Ruim & 41 & 134 & 24 & 76 & 15,7 & 16 & 55 & 12,5 & \\
\hline Muito ruim & 13 & 45 & 10 & 36 & 7,5 & 3 & 8 & 1,8 & \\
\hline
\end{tabular}

REAS/EJCH | Vol.12(8) | e3434 | DOI: https://doi.org/10.25248/reas.e3434.2020 Página 7 de 17 


\begin{tabular}{|c|c|c|c|c|c|c|c|c|c|}
\hline Percepção de saúde & & & & & & & & & \\
\hline Satisfatória & 108 & 330 & 55 & 165 & 34,2 & 52 & 163 & 37,0 & \multirow{2}{*}{0,755} \\
\hline Insatisfatória & 185 & 603 & 97 & 318 & 65,8 & 85 & 277 & 63,0 & \\
\hline \multicolumn{10}{|l|}{ Percepção de saúde bucal } \\
\hline Satisfatória & 163 & 330 & 85 & 260 & 55,1 & 76 & 256 & 59,0 & \multirow{2}{*}{0,236} \\
\hline Não satisfatória & 123 & 603 & 63 & 212 & 44,9 & 59 & 178 & 41,0 & \\
\hline \multicolumn{10}{|l|}{ Déficit cognitivo ${ }^{f}$} \\
\hline Não & 167 & 522 & 85 & 270 & 56,7 & 81 & 249 & 59,9 & \multirow{2}{*}{0,344} \\
\hline Sim & 119 & 390 & 63 & 206 & 43,3 & 51 & 167 & 40,1 & \\
\hline \multicolumn{10}{|l|}{ Incapacidade Funcionalg } \\
\hline Não & 214 & 666 & 106 & 325 & 66,6 & 106 & 335 & 79,4 & \multirow{2}{*}{0,000} \\
\hline Sim & 81 & 270 & 47 & 163 & 33,4 & 28 & 87 & 20,6 & \\
\hline \multicolumn{10}{|c|}{ História de queda no último ano } \\
\hline Não & 166 & 529 & 72 & 259 & 53,1 & 54 & 256 & 58,9 & \multirow{2}{*}{0,078} \\
\hline Sim & 128 & 413 & 81 & 229 & 46,9 & 81 & 179 & 41,1 & \\
\hline \multicolumn{10}{|l|}{ Polifarmáciah $^{h}$} \\
\hline Não & 216 & 685 & 119 & 373 & 76,3 & 93 & 290 & 67,7 & \multirow{2}{*}{0,004} \\
\hline Sim & 81 & 266 & 34 & 116 & 23,7 & 44 & 142 & 32,3 & \\
\hline \multicolumn{10}{|l|}{ Escala de depressão geriátrica ${ }^{i}$} \\
\hline Sem risco para depressão & 67 & 229 & 25 & 86 & 18,3 & 41 & 141 & 33,3 & \multirow{2}{*}{0,000} \\
\hline Com risco para depressão & 217 & 670 & 123 & 383 & 81,7 & 92 & 282 & 66,7 & \\
\hline \multicolumn{10}{|l|}{ Morbidades autorreferidas } \\
\hline Não & 9 & 34 & 3 & 8 & 1,6 & 5 & 20 & 4,5 & \multirow{2}{*}{0,010} \\
\hline Sim & 287 & 914 & 150 & 481 & 98,4 & 132 & 420 & 95,5 & \\
\hline \multicolumn{10}{|c|}{ Número de morbidades autorreferidas } \\
\hline Nenhuma & 9 & 34 & 3 & 8 & 1,6 & 5 & 20 & 4,5 & \multirow{3}{*}{0,001} \\
\hline 1 a 3 & 178 & 547 & 89 & 267 & 54,6 & 86 & 273 & 61,9 & \\
\hline$\geq 4$ & 109 & 366 & 61 & 214 & 43,8 & 46 & 148 & 33,6 & \\
\hline
\end{tabular}

REAS/EJCH | Vol.12(8) | e3434 | DOI: https://doi.org/10.25248/reas.e3434.2020 Página 8 de 17 


\begin{tabular}{|c|c|c|c|c|c|c|c|c|c|}
\hline Doença de colu & & & & & & & & & \\
\hline Não & 117 & 380 & 59 & 178 & 36,5 & 56 & 194 & 44,1 & \multirow{2}{*}{0,018} \\
\hline Sim & 179 & 567 & 94 & 310 & 63,5 & 81 & 246 & 55,9 & \\
\hline \multicolumn{10}{|c|}{ Hipertensão } \\
\hline Não & 81 & 256 & 42 & 130 & 26,7 & 36 & 115 & 26,1 & \multirow{2}{*}{0,833} \\
\hline Sim & 214 & 689 & 110 & 356 & 73,3 & 101 & 325 & 73,9 & \\
\hline \multicolumn{10}{|c|}{ Reumatismo, artrite, artrose } \\
\hline Não & 169 & 534 & 89 & 274 & 56,0 & 76 & 246 & 55,9 & \multirow{2}{*}{0,970} \\
\hline Sim & 127 & 414 & 64 & 215 & 44,0 & 61 & 194 & 44,1 & \\
\hline \multicolumn{10}{|c|}{ Doença do coração/cardiovascular } \\
\hline Não & 214 & 675 & 112 & 351 & 71,8 & 8 & 311 & 70,7 & \multirow{2}{*}{0,712} \\
\hline Sim & 82 & 272 & 41 & 138 & 28,2 & 39 & 129 & 29,3 & \\
\hline \multicolumn{10}{|c|}{ Osteoporose } \\
\hline Não & 217 & 697 & 112 & 351 & 71,8 & 100 & 329 & 74,8 & \multirow{2}{*}{0,335} \\
\hline Sim & 79 & 151 & 41 & 138 & 28,2 & 37 & 111 & 25,2 & \\
\hline \multicolumn{10}{|c|}{ Depressão } \\
\hline Não & 237 & 766 & 76 & 367 & 75,1 & 94 & 385 & 87,5 & \multirow{2}{*}{0,000} \\
\hline Sim & 57 & 176 & 77 & 122 & 24,9 & 43 & 55 & 12,5 & \\
\hline \multicolumn{10}{|c|}{ Diabetes } \\
\hline Não & 227 & 713 & 122 & 371 & 75,9 & 100 & 326 & 74,1 & \multirow{2}{*}{0,532} \\
\hline $\operatorname{Sim}$ & 69 & 234 & 31 & 118 & 24,1 & 37 & 114 & 25,9 & \\
\hline \multicolumn{10}{|c|}{ Câncer } \\
\hline Não & 274 & 880 & 138 & 443 & 90,6 & 131 & 421 & 95,7 & \multirow{2}{*}{0,002} \\
\hline Sim & 22 & 67 & 15 & 46 & 9,4 & 6 & 19 & 4,3 & \\
\hline \multicolumn{10}{|c|}{ Síndrome de Fragilidade ${ }^{j}$} \\
\hline Não & 193 & 614 & 87 & 209 & 51,0 & 106 & 405 & 78,2 & \multirow{2}{*}{0,000} \\
\hline Sim & 97 & 314 & 66 & 201 & 49,0 & 31 & 113 & 22,3 & \\
\hline
\end{tabular}

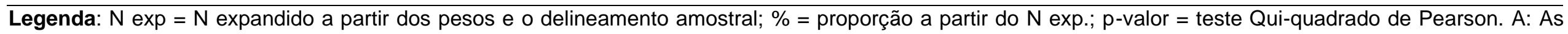

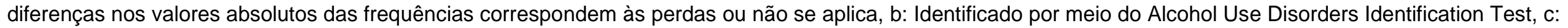

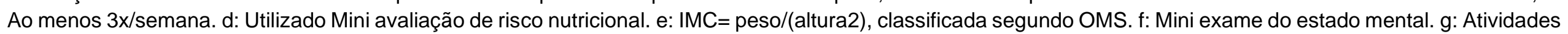

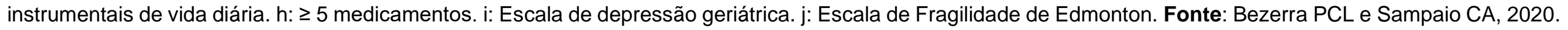


Tabela 2 - Análise bivariada das variáveis sociodemográficas, de estilo de vida e saúde da amostra com o risco de violência. Rio Branco, Acre. Idosos, 2016 - 2017.

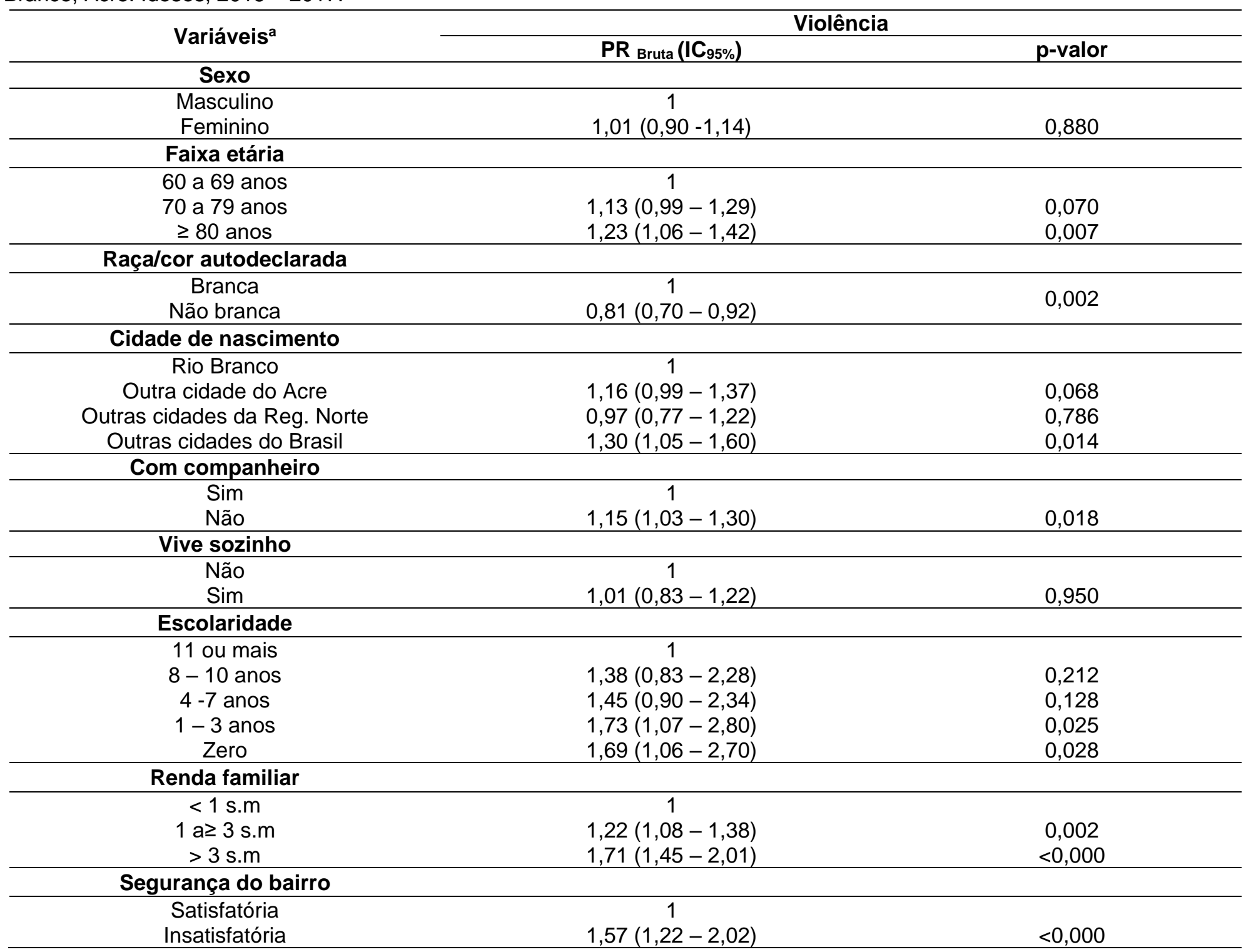

REAS/EJCH | Vol.12(8) | e3434 | DOI: https://doi.org/10.25248/reas.e3434.2020 Página 10 de 17 
Revista Eletrônica Acervo Saúde / Electronic Journal Collection Health ｜ ISSN 2178-2091

\begin{tabular}{|c|c|c|}
\hline \multirow{2}{*}{ Variáveis $^{\mathrm{a}}$} & \multicolumn{2}{|c|}{ Violência } \\
\hline & $\mathbf{P R}_{\text {Bruta }}\left(\mathbf{I C}_{95 \%}\right)$ & p-valor \\
\hline \multicolumn{3}{|l|}{ Hábito tabagista } \\
\hline Não & 1 & \\
\hline Sim & $1,11(0,97-1,26)$ & 0,138 \\
\hline \multicolumn{3}{|c|}{ Excesso de bebida alcóolica ${ }^{b}$} \\
\hline Não & 1 & \\
\hline Sim & $1,17(0,96-1,43)$ & 0,123 \\
\hline \multicolumn{3}{|c|}{ Prática de atividade físicac } \\
\hline $\operatorname{Sim}$ & 1 & \\
\hline Não & $1,25(1,07-1,47)$ & 0,006 \\
\hline \multicolumn{3}{|l|}{ Risco nutricional $^{d}$} \\
\hline Não & 1 & \\
\hline Sim & $0,96(0,85-1,09)$ & 0,512 \\
\hline \multicolumn{3}{|c|}{ (2) } \\
\hline Eutrófico & 1 & \\
\hline Baixo peso & $1,26(0,92-1,72)$ & 0,153 \\
\hline Sobrepeso & $0,71(0,49-1,03)$ & 0,074 \\
\hline Obeso & $0,88(0,67-1,14)$ & 0,331 \\
\hline \multicolumn{3}{|c|}{ Estado de saúde autorreferida } \\
\hline Muito bom & 1 & \\
\hline Bom & $2,33(1,30-4,16)$ & 0,004 \\
\hline Regular & $2,12(1,19-3,80)$ & 0,011 \\
\hline Ruim & $2,53(1,40-4,56)$ & 0,002 \\
\hline Muito ruim & $3,52(1,95-6,35)$ & $<0,000$ \\
\hline \multicolumn{3}{|c|}{ Percepção de saúde } \\
\hline Satisfatória & 1 & \\
\hline Insatisfatória & $1,10(0,90-1,33)$ & 0,359 \\
\hline \multicolumn{3}{|c|}{ Percepcão de saúde bucal } \\
\hline Satisfatória & 1 & \\
\hline Não satisfatória & $1,06(0,94-1,19)$ & 0,338 \\
\hline \multicolumn{3}{|l|}{ Déficit cognitivo $f$} \\
\hline Não & 1 & \\
\hline Sim & $1,06(0,95-1,20)$ & 0,303 \\
\hline \multicolumn{3}{|c|}{ Incapacidade Funcional ${ }^{9}$} \\
\hline Não & 1 & $<0000$ \\
\hline Sim & $1,31(1,17-1,47)$ & $<0,000$ \\
\hline
\end{tabular}

REAS/EJCH | Vol.12(8) | e3434 | DOI: https://doi.org/10.25248/reas.e3434.2020 Página 11 de 17 
Revista Eletrônica Acervo Saúde / Electronic Journal Collection Health ｜ ISSN 2178-2091

\begin{tabular}{|c|c|c|}
\hline \multirow{2}{*}{ Variáveis $^{\mathrm{a}}$} & \multicolumn{2}{|c|}{ Violência } \\
\hline & $\mathbf{P R}_{\text {Bruta }}\left(\mathbf{I C}_{95 \%}\right)$ & p-valor \\
\hline \multicolumn{3}{|c|}{ História de queda no último ano } \\
\hline Não & 1 & \\
\hline Sim & $1,12(1,00-1,26)$ & 0,052 \\
\hline \multicolumn{3}{|l|}{ Polifarmáciah $^{\mathrm{h}}$} \\
\hline Não & 1 & \\
\hline Sim & $0,80(0,69-0,93)$ & 0,003 \\
\hline \multicolumn{3}{|c|}{ Escala de depressão geriátrica ${ }^{i}$} \\
\hline Sem depressão & 1 & \\
\hline Com depressão & $1,52(1,28-1,81)$ & $<0,000$ \\
\hline \multicolumn{3}{|c|}{ Morbidades autorreferidas } \\
\hline Não & 1 & \\
\hline Sim & $1,78(1,03-3,08)$ & 0,040 \\
\hline \multicolumn{3}{|c|}{ Número de morbidades autorreferidas } \\
\hline Nenhuma & 1 & \\
\hline 1 a 3 & $1,66(0,95-2,90)$ & 0,073 \\
\hline$\geq 4$ & $1,96(1,13-3,41)$ & 0,017 \\
\hline \multicolumn{3}{|c|}{ Doença de coluna/costas } \\
\hline Não & 1 & \\
\hline Sim & $1,14(1,01-1,29)$ & 0,035 \\
\hline \multicolumn{3}{|l|}{ Hipertensão } \\
\hline Não & 1 & \\
\hline Sim & $0,98(0,86-1,12)$ & 0,750 \\
\hline \multicolumn{3}{|c|}{ Reumatismo, artrite, artrose } \\
\hline Não & 1 & \\
\hline Sim & $0,99(0,88-1,11)$ & 0,866 \\
\hline \multicolumn{3}{|c|}{ Doença do coração/cardiovascular } \\
\hline Não & 1 & \\
\hline Sim & $0,97(0,85-1,11)$ & 0,659 \\
\hline \multicolumn{3}{|l|}{ Osteoporose } \\
\hline Não & 1 & \\
\hline Sim & $1,06(0,93-1,21)$ & 0,367 \\
\hline \multicolumn{3}{|l|}{ Depressão } \\
\hline Não & 1 & \\
\hline Sim & $1,42(1,26-1,60)$ & $<0,000$ \\
\hline
\end{tabular}

REAS/EJCH | Vol.12(8) | e3434 | DOI: https://doi.org/10.25248/reas.e3434.2020 Página 12 de 17 
Revista Eletrônica Acervo Saúde / Electronic Journal Collection Health ｜ ISSN 2178-2091

\begin{tabular}{|c|c|c|}
\hline \multirow{2}{*}{ Variáveis $^{\mathrm{a}}$} & \multicolumn{2}{|c|}{ Violência } \\
\hline & $\mathbf{P R}_{\text {Bruta }}\left(\mathbf{I C}_{95 \%}\right)$ & p-valor \\
\hline \multicolumn{3}{|l|}{ Diabetes } \\
\hline Não & 1 & \\
\hline Sim & $0,93(0,81-1,08)$ & 0,341 \\
\hline \multicolumn{3}{|l|}{ Câncer } \\
\hline Não & 1 & \\
\hline Sim & $1,38(1,18-1,62)$ & $<0,000$ \\
\hline \multicolumn{3}{|l|}{ Derrame/AVC } \\
\hline Não & 1 & \\
\hline Sim & $1,26(1,09-1,46)$ & 0,002 \\
\hline \multicolumn{3}{|l|}{ Cirrose } \\
\hline Não & 1 & \\
\hline Sim & $1,14(0,75-1,73)$ & 0,533 \\
\hline \multicolumn{3}{|l|}{ Insuficiência renal crônica } \\
\hline Não & 1 & \\
\hline Sim & $0,8(0,70-1,10)$ & 0,260 \\
\hline \multicolumn{3}{|l|}{ Síndrome de Fragilidade } \\
\hline Não & 1 & \\
\hline Sim & $1,55(1,38-1,73)$ & $<0,000$ \\
\hline \multicolumn{3}{|l|}{ Síndrome de Fragilidade $\mathrm{j}$} \\
\hline Não tem & 1 & \\
\hline Aparentemente vulnerável & $0,89(0,73-1,07)$ & 0,210 \\
\hline Fragilidade leve & $1,43(1,23-1,66)$ & $<0,000$ \\
\hline Fragilidade moderada & $1,43(1,21-1,68)$ & $<0,000$ \\
\hline Fragilidade severa & $1,72(1,47-2,00)$ & $<0,000$ \\
\hline
\end{tabular}

Legenda: $p$-valor = teste Qui-quadrado de Pearson. ${ }^{a}$ As diferenças nos valores absolutos das frequências correspondem às perdas ou não se aplica. ${ }^{b}$ Identificado por meio do Alcohol Use Disorders Identification Test. ${ }^{c}$ Ao menos $3 \mathrm{x} /$ semana. ${ }^{d}$ Utilizado Mini avaliação de risco nutricional. ${ }^{4} \mathrm{IMC}=$ peso/(altura $\left.{ }^{2}\right)$, classificada segundo $\mathrm{OMS}$. ${ }^{\dagger}$ Mini exame do estado mental. $\mathrm{g}$ Atividades instrumentais de vida diária. ${ }^{h} \geq 5$ medicamentos. ' Escala de depressão geriátrica. j Escala de Fragilidade de Edmonton.

Fonte: Bezerra PCL e Sampaio CA, 2020. 
Os idosos que não praticam atividade física apresentaram maior prevalência de sofrer violência $(1,25$ IC $95 \%$ $1,07-1,47)$. Idosos que autorreferiram estado de saúde muito ruim apresentaram três vezes mais prevalência de violência 3,52 (IC95\% 1,95 - 6,35). Os com incapacidade funcional apresentaram maior prevalência de sofrer violência 1,31 ( $\mathrm{IC}_{95 \%}$ 1,17 - 1,47). Aqueles que com risco de depressão apresentaram maior prevalência de violência 1,52 (IC95\% 1,28-1,81), assim como os idosos que autorreferiram ter alguma morbidade $(1,78$ $\left.I_{\text {I }} 95 \% 1,03-3,08\right)$ e os que apresentaram algum grau de fragilidade $(1,55$ (C95\% 1,38-1,73). (Tabela 2).

Os idosos com quatro ou mais morbidade autorreferidas apresentaram maior prevalência de sofrer violência 1,96 (IC95\% 1,13-3,41). Assim também os que tem doenças de coluna/costas com de maior prevalência de sofrer violência 1,14 (IC95\% 1,01 - 1,29). Aqueles com depressão apresentam maior prevalência de sofrer violência 1,42 (IC95\% 1,26 - 1,60). Idosos com câncer tem maior prevalência de sofrer algum tipo de violência 1,38 (IC95\% 1,38 - 1,62).

Aqueles que tiveram Derrame/AVC tem maior prevalência de sofrer violência 1,26 (IC95\% 1,09 - 1,46) (Tabela 2). Mantiveram-se no modelo descritivo de associação com a prevalência de violência geral nos idosos da amostra (Tabela 3), as variáveis: segurança do bairro; renda familiar; síndrome de fragilidade, depressão; história de câncer; acidente vascular cerebral, etnia/cor e consumo excessivo de álcool, sendo as variáveis que expressam pior situação de saúde foram a maioria.

Tabela 3 - Análise multivariada das variáveis selecionadas para o modelo descritivo de associação com a prevalência de violência. Rio Branco, Acre. Idosos, 2016 - 2017.

\begin{tabular}{|c|c|c|}
\hline Variáveis & $\begin{array}{c}\text { Violência } \\
\text { PR }\end{array}$ & p-valor \\
\hline \multicolumn{3}{|c|}{ Segurança do bairro } \\
\hline $\begin{array}{c}\text { Satisfatória } \\
\text { Insatisfatória }\end{array}$ & $\begin{array}{c}1 \\
1,46(1,15-1,86)\end{array}$ & 0,002 \\
\hline \multicolumn{3}{|l|}{ Renda Familiar } \\
\hline $\begin{array}{c}<1 \mathrm{~s} . \mathrm{m} \\
1 \mathrm{a}<3 \mathrm{~s} . \mathrm{m} \\
\geq 3 \text { s.m }\end{array}$ & $\begin{array}{c}1 \\
1,12(0,98-1,27) \\
1,41(1,22-1,64)\end{array}$ & $\begin{array}{r}0,093 \\
<0,001\end{array}$ \\
\hline \multicolumn{3}{|c|}{ Síndrome de Fragilidade ${ }^{a}$} \\
\hline $\begin{array}{l}\text { Não } \\
\text { Sim }\end{array}$ & $\begin{array}{c}1 \\
1,41(1,26-1,57)\end{array}$ & $<0,001$ \\
\hline \multicolumn{3}{|l|}{ Depressão } \\
\hline $\begin{array}{l}\text { Não } \\
\text { Sim }\end{array}$ & $\begin{array}{c}1 \\
1,40(1,24-1,57)\end{array}$ & $<0,001$ \\
\hline \multicolumn{3}{|l|}{ Câncer } \\
\hline $\begin{array}{l}\text { Não } \\
\text { Sim }\end{array}$ & $\begin{array}{c}1 \\
1,20(1,02-1,41)\end{array}$ & 0,029 \\
\hline \multicolumn{3}{|c|}{ Acidente Vascular Cerebral } \\
\hline $\begin{array}{l}\text { Não } \\
\text { Sim }\end{array}$ & $\begin{array}{c}1 \\
1,20(1,03-1,40)\end{array}$ & 0,021 \\
\hline \multicolumn{3}{|l|}{ Etnia/cor } \\
\hline $\begin{array}{c}\text { Não branca } \\
\text { Branca }\end{array}$ & $\begin{array}{c}1 \\
1,17(1,03-1,34) \\
\end{array}$ & 0,016 \\
\hline \multicolumn{3}{|c|}{ Excesso de bebida alcóolicab } \\
\hline $\begin{array}{l}\text { Não } \\
\text { Sim }\end{array}$ & $\begin{array}{c}1 \\
1,31(1,08-1,59)\end{array}$ & 0,006 \\
\hline
\end{tabular}




\section{DISCUSSÃO}

Os resultados obtidos na análise dos dados deste estudo apontaram que, dentre os tipos de violência investigados, a violência mais frequente relatada a psicológica, seguida da violência financeira e, por último, porém com uma prevalência expressiva de $17,2 \%$, a violência física.

Observou-se a semelhança da prevalência encontrada na pesquisa, com a verificada em outros estados brasileiros, considerando a caraterização da violência nas suas várias dimensões o que reforça ainda mais a importância de pesquisa nessa área (MASCARENHAS MDM, et al., 2012). Em uma análise realizada no Município de Uberaba, Minas Gerais, a violência psicológica foi mais prevalente com $20,9 \%(n=152)$ sob a violência física que foi de $5,9 \%(n=43)$ (PAIVA MM e TAVARES DMS, 2015).

Segundo Bolsoni CC, et al. (2012), a violência psicológica faz com que aumentem as chances de ocorrência de outros tipos de violência contra a pessoa idosa, haja vista que os idosos ficam mais vulneráveis às situações de humilhação, medo e ações negativas causadas pelo agressor. Dessa forma, a alta prevalência de violência psicológica encontrada na amostra $(30,6 \%)$, além de já constituir uma agrave situação de violação humana, pode ser premissa para outras formas de violência da vítima.

A violência financeira foi a segunda mais prevalente na amostra, geralmente, ocorre de modo cumulativo com outros tipos de violência contra o idoso. Possivelmente, é mais presente no convívio da sociedade brasileira do que mostram as estatísticas, visto que o idoso vítima desse tipo de violência, bem como de outros tipos, apresenta sentimento de culpa, vergonha e/ou medo causado por parte do agressor (SANTOS RC, et al., 2019), fatores limitantes para a denúncia. Em um estudo realizado em uma capital do Nordeste foram analisados 555 Boletins de Ocorrência na delegacia de Segurança e Proteção ao Idoso, destes 327, que representam $58,9 \%$, corresponderam-se à violência financeira que foi mais prevalente comparada com os outros tipos de violência (SANTOS AMR, et al., 2019).

A prevalência de violência física apresentada no estudo (17,2\%) mostrou-se acima dos dados encontrados com idosos de uma Capital do Nordeste, que apresentou a prevalência de $11 \%$ de violência física (SANTOS AMR, et al., 2019). A violência física ganha destaque pelo fato de produzir diversas consequências visivelmente graves ao idoso, como: o sofrimento; as contusões; o medo; a depressão; a diminuição da qualidade de vida, danos ou infração dos direitos humanos, (CORREIA TMP, et al., 2012).

$\mathrm{O}$ modelo descritivo identificou os fatores associados à prevalência de violência geral na amostra estudada. Os idosos que relataram insegurança de bairro foram os que mais sofrem violência geral. Entendese este fator, revela a gravidade de um problema de segurança pública, que levaria os idosos a ficarem mais limitados ao ambiente domiciliar, favorecendo ao sedentarismo, o que pode aumentar as chances de morbidades, dependências e à violência domiciliar. Um estudo realizado em Buenos Aires aponta que o medo do crime se transforma em um problema bem maior do que o próprio delito, quando o medo da criminalidade, diferentemente da real criminalidade, tem afetado de maneira grandiosa os indivíduos, com consequências perene e rude (VARELA C, 2005).

Sabe-se através de uma pesquisa realizada em uma Capital do Nordeste, que a renda e os bens da pessoa idosa são os principais motivos geradores de abuso e maus-tratos contra idosos, (SANTOS AMR et al., 2019). No atual estudo, a maioria das pessoas entrevistadas que relatou possuir renda maior ou igual a três salários mínimos, apresentou maior associação com a prevalência de violência. Para alguns pesquisadores a questão da dependência econômica de filhos adultos em relação a pais idosos é muito presente no Brasil, e consiste em fator de risco para violência, especialmente quando o idoso é a única fonte de recursos da família (MINAYO MC, 2004).

A prevalência de violência contra os idosos investigados que referiram ser da cor/etnia banca foi superior à observada em uma pesquisa em São Paulo, que analisou 712 denúncias de maus tratos contra idosos pelo Dick 100, entre 2003 até 2009, onde se verificou que houve mais idosos vítimas de violência, no grupo de etnia/cor preta (PINTO FNFR, et al., 2013).

No entanto, acredita-se que esta diferença com relação aos dados encontrados na amostra, seja em decorrência do tipo de violência, majoritariamente, identificado no estudo (violência psicológica e financeira) e o corriqueiramente, denunciado (negligência, abandono e física). 
Algumas pesquisas trazem dados do perfil do agressor. Na cidade de Caruaru, Estado de Pernambuco, mostrou que a maioria dos agressores de idosos são os filhos com $(47,6 \%)$, depois o cônjuge (16,7\%) (LOPES LFG, et al., 2015). Outro estudo realizado na cidade de Recife, que tinha como tema violência doméstica contra o idoso, trouxe dados semelhantes, apontando os filhos como principal agressor com $(46,15 \%)$, sendo que a maioria fazia uso de bebida alcoólica (SILVA CFS e DIAS CMSB, 2015). Chama-se a atenção que o excesso de bebida alcoólica, além de estar associado ao perfil do agressor, como mostra a literatura, também se apresentou como um fator de associação nos idosos vítimas de violência na amostra do estudo.

Os idosos da pesquisa com síndrome de fragilidade apresentaram $41 \%$ mais prevalência de violência, comparados aos idosos robustos. Semelhantemente, uma pesquisa realizada na cidade de Brasília, $70 \%$ dos idosos com síndrome de fragilidade sofreram mais violência, quando comparado aos idosos robustos (FAUSTINO AM, et al., 2014). Existe a evidência de que idosos dependentes são mais vulneráveis à ocorrência de violência física (REIS LA, et al., 2014).

Nesse estudo, os idosos com depressão foram os que sofreram mais violência, de maneira similar com o que acontece em idosos de outras localidades do mundo. Na China, a chance de ocorrência de maus-tratos foi 5,5 vezes maior em 2.039 idosos deprimidos, comparados aos não deprimidos, que moravam em comunidades da zona rural (WU L, et al., 2012). No Brasil, algumas pesquisas evidenciam a associação de violência com depressão e outras condições geradoras de vulnerabilidade em longevos (APRATTO JÚNIOR PC, 2010).

Aqueles que relataram história de câncer e/ou acidente vascular cerebral, foram os que apresentaram maior prevalência de violência. Possivelmente, as limitações geradas, nestas condições, geram impactos consideráveis na vida do idoso e de seu cuidador e ou familiar. Fica evidente que as piores condições de saúde estão associadas à ocorrência de violência. Podem estar relacionadas à maior dependência do idoso para realizar suas atividades de vida diária, redução da qualidade de vida e o estresse do cuidador (PEDREIRA LC e LOPES RLM, 2010).

A vulnerabilidade do idoso em sofrer violência soma-se a vários outros fatores. Dentre esses outros fatores, as causas mais frequentes estão relacionadas ao processo de fragilização vivenciado pelo idoso e ao alto nível de dependência familiar e/ou do cuidador (GARBIN CAS, et al., 2016).

\section{CONCLUSÃO}

Os resultados encontrados neste estudo, identificaram que fatores sociais e de saúde estão associados à prevalência de violência geral nos idosos investigados. No entanto, a maioria desses fatores foram referentes à uma saúde fragilizada (depressão, câncer, AVC, síndrome de fragilidade e excesso de consumo de álcool). Verificou, ainda, altas prevalências, segundo a tipologia da violência, sendo a violência psicológica a mais prevalente $(30,6 \%)$, seguida da violência financeira $(25 \%)$ e física $(17,2 \%)$. Quanto ao perfil social dos idosos associados à violência geral, destaca-se que foram os de renda familiar acima de três salários mínimos, etnia autodeclarada branca e os que relataram segurança do bairro insatisfatória que apresentaram maior associação. A pesquisa é relevante, pois traz subsídios que irão colaborar para os debates que tem como tema a violência contra o idoso, contribuindo para a identificação da magnitude do problema, do perfil de risco para rastreio e, possivelmente, para a proposição de ações necessárias à rede de cuidado do idoso vítima de violência.

\section{REFERÊNCIAS}

1. ALMEIDA OP, ALMEIDA SA. Confiabilidade da Versão Brasileira da Escala de Depressão em Geriatria (GDS) Versão Reduzida. Arquivo de Neuropsiquiatria, 1999; 57(2):421-426.

2. APRATTO JÚNIOR PC. The domestic violence against the elderly within the Family Health Program of Niterói (RJ, Brazil). Ciência \& saúde coletiva, 2010; 15(6): 2903-2995.

3. BABOR TF, et al. AUDIT-The Alcohol Use Disorders Identification Test: guidelines for use in primary care. 2nd Ed. Geneva: World Health Organization, 2001.

4. BERTOLUCCI PHF, et al. O Mini Exame do Estado Mental em uma população geral: impacto da escolaridade. Arq. Neuropsiquiatr, 1994; 52(1):1-7. 
5. BRASIL. Conselho Nacional de Saúde. Resolução n 466, 2012. Diretrizes e Normas regulamentadoras de pesquisa envolvendo seres humanos. Brasília, 13. jun. 2013. Seção 1 p. 59.

6. BOLSONI CC, et al. Prevalência de violência contra idosos e fatores associados, estudo de base populacional em Florionópolis, SC. Revista Brasileira de Geriatria e Gerontologia, 2016; 19(4):671-682.

7. CHAIMOWICZ F. A saúde dos idosos brasileiros às vésperas do século XXI: problemas, projeções e alternativas. Revista de Saúde Pública, 1997; 31(2): 184-200.

8. CORREIA TMP, et al. Perfil dos idosos em situação de violência atendidos em serviço de emergência em Recife-PE. Revista Brasileira de Geriatria e Gerontologia, 2012; 15(3): 529-536.

9. REIS LA, et al. Expressão da violência intrafamiliar contra idosos. ACTA Paulista de Enfermagem, 2014; 27(5): 434439.

10. FAUSTINO AM, et al. Capacidade funcional e situações de violência em idosos. ACTA Paulista de Enfermagem, $2014 ; 27(5):$ 392-398.

11. GARBIN CAS, et al. Elderly victims of abuse: a five year document analysis. Revista Brasileira de Geriatria e Gerontologia, 2016; 19(1): 87-94.

12. LOPES LGF, et al. Violência contra a pessoa idosa em um município do interior do estado de pernambuco. Anais do VI Congresso Internacional de Envelhecimento Humano, 2019 (1): 5-10. Disponível em:<http://www.editorarealize.com.br/revistas/cieh/trabalhos/TRABALHO_EV075_MD4_SA1_ID44_1610201701403 $0 . p d f>$. Acessado em 19 de novembro 2019.

13. GUIGOZ Y, et al. Mini Nutritional Assessment: A practical assessment tool for grading the nutritional state of elderly patients. Facts Res Gerontol,1994; 4(2):15-59.

14. KRUG EG, et al. World report on violence and health. Geneva: World Health Organization, 2002. MASCARENHAS MDM, et al. Violência contra a pessoa idosa: análise das notificações realizadas no setor saúde. Ciência \& Saúde Coletiva, 2012; 17(9): 2331-2341.

15. MATSUDO SM, et al. Questionário Internacional de Atividade Física (IPAQ): estudo de validade e reprodutibilidade no Brasil. Rev Bras Ativ Saude. 2001; 10:5-18.

16. MINAYO MC. Violência contra idosos: o avesso de respeito à experiência e à sabedoria. Cartilha da Secretaria Especial dos Direitos Humanos, 2004; 1-48.

17. NEALE AV, et al. Validation of the Hwalek-Sengstock Elder Abuse Screening Test. J Appl Gerontol, 1991; 10:406-18.

18. Organização Mundial De Saúde (OMS). World Health Organization. Physical status: the use and interpretation of anthropometry. Geneva: World Health Organization. 1995, Technical Report Series, p.854.

19. PAIVA MM, TAVARES DMS. Violência física e psicológica contra idosos: prevalência e fatores associados. Rvista Brasileira de Enfermagem, 2015; 68(6): 1035-1041.

20. PEDREIRA LC, LOPES RLM. Cuidados domiciliares ao idoso que sofreu Acidente Vascular Cerebral. Revista Brasileira de Enfermagem, 2010; 63(5): 837-840.

21. REICHENHEIM ME, et al. Adaptação transcultural para o português (Brasil) do instrumento Hwalek-Sengstock Elder Abuse Screening Test (H-S/EAST) utilizado para identificar risco de violência contra o idoso. Cad. Saúde Pública, $2008 ; 24(8): 1801-1813$.

22. ROLFSON DB, et al. Validity and reliability of the Edmonton Frail Scale. Age and ageing, 2006; 35(5):526-529. ROSA TEC, et al. Fatores determinantes da capacidade funcional entre idosos. Rev Saude Publica, 2003; 37(1):40-8.

23. SANTOS AMR, et al. Violência econômico-financeira e patrimonial contra o idoso: estudo documental. Rev. esc. enferm. USP [online]. 2019, 53.

24. SANTOS RC, et al. Violência e fragilidade na pessoa idosa. Revista de Enfermagem UFPE [online], 2018; 12(8): 2227.

25. SILVA CFS, DIAS CMSB. Violência contra idosos: perfil sociodemográfico dos familiares agressores, tipos de violência impetrada e motivações para sua ocorrência. Revista Eletronica Gestão \& Saúde, 2015; 7(2): 563.

26. VARELA C. Qué significa estar seguro: De delitos, miedos e inseguridades entre los adultos mayores. Cuadernos de Antropología Social, 2005; 2005(22): 153-171.

27. WU L, et al. Prevalence and associated factors of elder mistreatment in a rural community in people's republic of china: A cross-sectional study. PLoS ONE, 2012; 7(3).

28. YON Y, et al. Elder abuse prevalence in community settings: a systematic review and meta-analysis. The Lancet Global Health, 2017; 5(2):147-156. 